\title{
Zum Zusammenspiel von Recht und Ökonomik bei Effizienzanforderungen an Unternehmen
}

\author{
Michael Fehling* \\ Rezension zu Markus Ludwigs, Unternehmensbezogene Effizienzanforderungen im Öffentlichen \\ Recht. Unternehmenseffizienz als neue Rechtskategorie, Berlin (Duncker \& Humblot - \\ Das Öffentliche Recht, Habilitationen, Band 1) 2013, 674 Seiten, ISBN 978-3-428-13984-2
}

Fragen der Ökonomisierung beschäftigen das (öffentliche) Recht schon seit über 20 Jahren. Bislang hat man dabei Effizienzanforderungen meist mit Blick auf die staatliche Verwaltung diskutiert. Doch auch in der modernen Wirtschaftsregulierung nimmt das (Verwaltungs-)Recht immer öfter auf ökonomische Maßstäbe Bezug. Deshalb lenkt die vorliegende Habilitationsschrift, in Bonn bei Matthias SchmidtPreuß entstanden, den Blick auf rechtliche Effizienzanforderungen an Unternehmen. Als Referenzgebiete dienen die Regulierung der Netzwirtschaften, das im Entstehen begriffene Energieeffizienzrecht sowie das Kartellrecht. Dies verspricht Erkenntnisgewinn auf drei Ebenen: grundlegend-theoretisch zur Transformation außerrechtlicher (ökonomischer) in juristische Maßstäbe, systembildend zu Möglichkeiten und Grenzen rechtsgebietsübergreifender Kohärenz sowie rechtsdogmatisch schließlich sogar für die eine oder andere konkrete Auslegungsfrage.

1. In einem ersten, den Grundlagen gewidmeten Teil steht am Anfang eine konzise und zugleich sehr inhaltsreiche Aufarbeitung der maßgeblichen betriebswirtschaftlichen und vor allem volkswirtschaftlichen Theorie. Bei den Effizienzkonzepten konzentriert sich die Darstellung auf die allokative Effizienz (Stichworte: Pareto-Optimum, Kaldor-Hicks-Kriterium), aber auch produktive sowie dynamische Effizienz und die problematischen Wechselbeziehungen werden angesprochen. Im Rahmen der normativen Theorie der Regulierung werden die Bedeutung von natürlichen Monopolen, externen Effekten und Informationsasymmetrien einprägsam herausgearbeitet. Wenngleich die herrschenden Anschauungen in der Ökonomie im Vordergrund stehen, deutet Ludwigs doch auch immer wieder geschickt Kritikpunkte und alternative Ansätze an. Etwas blasser bleibt der anschließende Überblick über die herkömmliche staatsgerichtete Effizienzperspektive mit kurzer Diskussion eines verfassungsrechtlichen Wirtschaftlichkeitsgebots und verwaltungsrechtlicher Effizienzkonzepte in Form vor allem des Neuen Steuerungsmodells und der Beschleunigungsgesetzgebung. Formal bereits zum zweiten Teil, der Sache nach aber noch zu den Grundlagen gehört dann der Perspektivenwechsel hin zu unternehmensbezogenen Effizienzkonzepten. Überraschend knapp fällt dabei der Blick auf die Einbruchstellen für ökonomische unternehmensbezogene Effizienzanforderungen in das Recht aus.

* Prof. Dr. Michael Fehling, LL.M. (Berkeley), hat einen Lehrstuhl für Öffentliches Recht mit Rechtsvergleichung an der Bucerius Law School. Hamburg, inne. 
Ludwigs unterscheidet die direkte Rezeption im Gesetz, die Ausfüllung rechtlicher Generalklauseln und die Verankerung allgemeiner Zielvorstellungen. Er betont dabei besonders, dass sich der „Gestaltwandel von einem ökonomischen in ein juristisches Konzept" ausschließlich nach den Regeln des Rechts vollziehen muss. Dies wird in der folgenden Analyse nicht zuletzt in der Aufgabe sichtbar, die rechtlichen Effizienzanforderungen grundrechtskonform zu interpretieren.

Der zweite Teil untersucht danach das Verständnis von Unternehmenseffizienz in drei Referenzgebieten. Es beginnt mit dem Regulierungsrecht und dort wiederum mit der Regulierung der Zugangsentgelte in den Netzwirtschaften. Die „Kosten effizienter Leistungserbringung “ in der Telekommunikation und die Umstellung auf die (Effizienz-)Anreizregulierung im Energiewirtschaftsrecht stehen im Vordergrund und werden zum Vollkostenansatz mit nur sehr beschränkten zusätzlichen Effizienzanforderungen im Eisenbahnsektor abgegrenzt. Hier wie auch in der Folge knüpft Ludwigs immer wieder souverän an den ökonomischen Eingangsteil an. Als weitere Konstellation aus dem Regulierungsrecht wird die Frequenzvergabe in der Telekommunikation mit Schwerpunkt auf dem Versteigerungsverfahren thematisiert. Man hofft, damit die effizientesten Nutzer der knappen Frequenzen anhand ihrer Zahlungsbereitschaft zu identifizieren. Als zweites Referenzgebiet dient das Energieumweltrecht mit der Forderung nach Energieeffizienz. Nach erneuter Vertiefung der hier relevanten ökonomischen Grundlagen spannt sich der Bogen von der Energieverbrauchskennzeichnung über den Emissionshandel bis zu Energieeffizienzanforderungen an immissionsschutzrechtlich genehmigungsbedürftige Anlagen. Angesichts der Heterogenität dieser Regelungskomplexe soll die Steuerungsperspektive eine Systematisierung des anzutreffenden Instrumentenmixes liefern. Das dritte und letzte Referenzgebiet ist das (europäische) Kartellrecht mit Kartellverbot, Missbrauchsverbot und Zusammenschlusskontrolle. Die Kontroverse um einen „more economic approach “ konzentriert sich dabei auf eine Effizienzverteidigung für eigentlich kartellrechtswidrige Verhaltensweisen. Letztlich folge das Recht fast durchweg einem Konsumentenwohlfahrts- und nicht dem in der Ökonomie präferierten Gesamtwohlfahrtsstandard.

Den Abschluss bildet die formal noch zum zweiten Teil gehörende systematisierende Synthese. Ludwigs identifiziert Interdisziplinarität, Multifunktionalität, Multifinalität und Grundrechtsfestigkeit als prägende Strukturmerkmale der Unternehmenseffizienz im Recht. Unter dem Stichwort Interdisziplinarität werden die unterschiedlichen Einbruchsstellen ökonomischer (Unternehmens-)Effizienzkonzepte in das Recht zusammengestellt: von der direkten Rezeption des Effizienzbegriffs in den Tatbestandsmerkmalen einer Norm über die Ausfüllung von Generalklauseln bis hin zur ausdrücklichen oder konkludenten Verankerung als auslegungsleitende allgemeine Zielvorstellung des Gesetzes. Die Multifunktionalität der Unternehmenseffi- 
zienz offenbare sich in ihrem Charakter als Rechtspflicht, Genehmigungsvoraussetzung, Anreiz, Auswahl- und Verteilungskriterium oder Einwand, oftmals auch kombiniert. Multifinal wirkten solche Effizienzanforderungen insoweit, als sie kein Selbstzweck sein könnten, sondern ihrerseits wiederum ganz unterschiedlichen Gemeinwohlzielen dienten. Die prima facie wohlfahrtsfördernde Steigerung volkswirtschaftlicher Allokationseffizienz stehe oft im Vordergrund, doch könne die „Politik des Gesetzes“ auch auf primär außerökonomische Ziele wie den Umwelt- und Klimaschutz oder die Versorgungssicherheit ausgerichtet sein. Zur Sicherung der Grundrechtsfestigkeit dürften die Effizienzanforderungen nicht auf ein ökonomischtheoretisch definiertes Optimum ausgerichtet werden, sondern müssten für die Unternehmen mit zumutbarem Aufwand erfüllbar bleiben; die maßgeblichen Vorschriften seien jedoch durchweg entsprechend verfassungskonform auslegbar.

2. Die große Stärke dieser Habilitationsschrift liegt in ihrer Systematisierungskraft. Souverän führt Ludwigs den Leser durch die unterschiedlichsten Rechtsgebiete, dabei immer wieder gekonnt ökonomische und juristische Aspekte verknüpfend. Der Stil ist gänzlich unprätentiös, doch offenbart sich darin die seltene Gabe, schwierige Zusammenhänge in einer gelungenen Balance zwischen Differenzierung und notwendiger Komplexitätsreduzierung deutlich zu machen. Schon der ökonomische Grundlagenteil ist insoweit eine besondere Leistung, als darin eine Vielzahl von Ansätzen zu einem schlüssigen Gesamtbild zusammengefügt wird, ohne inhärente Spannungen und Kontroversen zu verdecken. Mögen auch viele rechtliche Einzelergebnisse wenig spektakulär sein, so gelingt es Ludwigs doch immer wieder, das Verständnis der Rechtslage einschließlich vieler Streitfragen in den klug gewählten Referenzgebieten deutlich zu vertiefen. Vor allem fördert der neue Blickwinkel manche verborgenen Zusammenhänge zu Tage.

Etwas blasser bleibt die Arbeit dagegen in der Analyse der Mechanismen für die rechtliche Verarbeitung ökonomischer Effizienzkonzepte. Zwar überzeugt auch hier der systematisierende Ansatz und der Hinweis auf das Primat des Rechts, doch wäre eine weitere Vertiefung und Problematisierung wünschenswert gewesen. So könnte man z.B. überlegen, inwieweit sich ein etwaiger Meinungswandel in der Ökonomie oder sonstige neue Erkenntnisse in einer dynamischen Auslegung und Anwendung rechtlicher Scharnierbegriffe abbilden lassen. In Folge der Wirtschaftskrise ist ohnehin die Skepsis gegenüber vermeintlich gesicherten ökonomischen Erkenntnissen gewachsen und die Beschränktheit des Wissens zunehmend ins Bewusstsein gerückt. Inwieweit sollte sich dies, so ließe sich noch weitergehend fragen, auch in einer $\mathrm{Zu}$ rückdrängung holistischer ökonomischer Effizienzkonzepte bei der Auslegung von rechtlichen Anforderungen an Unternehmen niederschlagen? Die Arbeit weist durchaus an verschiedenen Stellen auf Wissensdefizite und (notwendige) Unvollkommenheiten in der Wirklichkeit hin, etwa in Zusammenhang mit der ökonomischen Theo- 
rie des „second best“, in der Erwähnung von Transaktionskosten oder bei den immerhin angedeuteten Funktionsschwächen des derzeitigen Emissionshandelssystems. Doch werden daraus - aus Sicht des Rezensenten - zu wenig Konsequenzen gezogen. So plädiert Ludwigs z.B. für eine gerichtliche Vollkontrolle von (Entgelt-)Regulierungsentscheidungen. Eine größere Skepsis gegenüber eindeutigen ökonomischen Ableitungen könnte bei solch hochkomplexen Regulierungsentscheidungen womöglich doch administrative Entscheidungsspielräume nahelegen.

Überzeugend zeigt Ludwigs an verschiedenen Stellen auf, dass Effizienzanforderungen an Unternehmen ökonomisch tendenziell auf Optimierung angelegt sind, juristisch aber ihre Grenze an der grundrechtlichen Verhältnismäßigkeit finden. Die Benchmark-Funktion des Standards der „Kosten effizienter Leistungserbringung“ sowie der Anreizregulierung und das Konzept des „front runner“ bei der Energieeffizienz von Produkten werden unter diesen beiden Aspekten gewürdigt. Insgesamt bleibt dabei aber das Effizienzkonzept tendenziell doch etwas statisch. Namentlich bei der Energieeffizienz hätte man über ambitioniertere Strategien eines „technology forcing " nachdenken können, wie es vor allem in den USA im Umweltrecht ausführlich diskutiert und für Auto-Abgasstandards in den 1970er Jahren auch praktiziert worden war. Effizienzanforderungen müssen, wenn sie hinreichend in die $\mathrm{Zu}$ kunft verlegt werden, nicht bereits heute technisch und wirtschaftlich erreichbar sein. Grundrechtlich könnten womöglich zusätzliche Spielräume für effizienzorientierte Regulierungsmaßnahmen daraus erwachsen, dass betroffene Unternehmenssparten zuvor von massiver staatlicher finanzieller Unterstützung profitiert haben. Die Arbeit lenkt richtigerweise den Blick darauf, dass Sonderrechte und staatliche Finanzierung beim Aufbau von Netzinfrastruktur zwar nicht den Grundrechtsschutz der Nachfolgunternehmen der früheren Staatsunternehmen beseitigen, wohl aber eine erhöhte Sozialbindung des (Netz-)Eigentums rechtfertigen können. Vielleicht ließe sich dieser Gedanke über die Netzsektoren hinaus sogar auf die massive Unterstützung vor allem von Unternehmen der Finanzbranche in der Wirtschaftskrise übertragen und könnte dort - ein weiteres möglicherweise lohnendes Referenzgebiet - veränderte Effizienzanforderungen bei den Rahmenbedingungen von Finanztransaktionen (etwa bezüglich der Eigenkapitalausstattung) legitimieren.

Hervorhebung verdient schließlich noch einmal die Erkenntnis, dass Unternehmenseffizienz nur Mittel oder allenfalls Zwischenziel sein kann, entsprechende die Unternehmen belastende Anforderungen daher durch andere Gemeinwohlziele gedeckt sein müssen. Geänderte Prioritäten können insoweit eine Umsteuerung nahelegen. So wird etwa derzeit über die Fortentwicklung (wenn nicht gar grundlegende Änderung) der Anreizregulierung im Energiesektor diskutiert, um dem zusätzlichen Investitionsbedarf infolge der Energiewende besser Rechnung zu tragen. In Effizienz- 
kategorien ausgedrückt könnte man darin eine Zurückdrängung der allokativen Effizienz zugunsten der Umwelteffizienz erblicken.

Mag man demnach auch über die eine oder andere Akzentsetzung streiten können (und müssen). Die Fruchtbarkeit der in dieser Arbeit eingenommenen Perspektive wird gerade daran deutlich. Dem selbst gestellten Anspruch, Unternehmenseffizienz als Rechtskategorie ins Bewusstsein zu rücken und in ihren vielen positivrechtlichen Facetten auszuleuchten, ist Markus Ludwigs mit diesem Werk voll gerecht geworden. 